\title{
Evaluation of satisfaction with a model of structured contraceptive counseling: Results from the LOWE trial
}

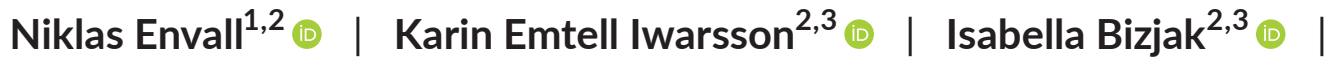 \\ Kristina Gemzell Danielsson ${ }^{2,3}$ — | Helena Kopp Kallner ${ }^{2,4,5}$ [
}

\author{
${ }^{1}$ School of Health and Welfare, Dalarna \\ University, Falun, Sweden \\ ${ }^{2}$ Department of Women's and Children's \\ Health, Karolinska Institutet, Stockholm, \\ Sweden \\ ${ }^{3}$ WHO Collaborating Centre, Division of \\ Gynecology and Reproduction, Karolinska \\ University Hospital, Stockholm, Sweden \\ ${ }^{4}$ Department of Obstetrics and \\ Gynecology, Danderyd Hospital, \\ Stockholm, Sweden \\ ${ }^{5}$ Department of Clinical Sciences at \\ Danderyd Hospital, Division of Obstetrics \\ and Gynecology, Karolinska Institutet, \\ Stockholm, Sweden \\ Correspondence \\ Niklas Envall, School of Health and \\ Welfare, Dalarna University, Högskolan \\ Dalarna, 79188 Falun, Sweden. \\ Email:nen@du.se \\ Funding information \\ This study has been partly funded by The \\ Swedish Research Council for Health, \\ Work-life and Welfare - FORTE, Grant \# \\ 2016-01063 and by The European Society \\ of Contraception and Reproductive \\ Health - ESC, Grant \# ESC P-2016-B-02 \\ and Karolinska Institutet and Region \\ Stockholm.
}

\begin{abstract}
Introduction: Intervention trials of structured contraceptive counseling have proved to increase use of long-acting reversible contraceptives (LARCs) and decrease numbers of unintended pregnancies. However, these interventions have not been evaluated from a user perspective. This study aimed to evaluate both healthcare providers' and participants' satisfaction with an intervention used in a large trial in Sweden.
\end{abstract}

Material and methods: A cross-sectional study on the intervention group from a cluster randomized trial conducted at 28 clinics in Stockholm, Sweden. Clinics were randomized (1:1 allocation ratio) to provide either structured contraceptive counseling (intervention) or standard contraceptive counseling (control). The intervention consisted of four parts; an educational video to be seen by the participant prior to contraceptive counseling, key questions to be asked by the healthcare provider, an effectiveness chart, and a box of contraceptive models. Eligible participants were 18 years or older, sexually active without a wish to conceive, and with the main purpose of contraceptive use being pregnancy prevention. Healthcare providers completed an electronic semi-structured survey to evaluate the intervention. This study analyses provider and participant satisfaction with the counseling material used in the intervention and if the intervention was found to be supportive in contraceptive counseling and contraceptive choice. Trial registration: ClinicalTrials.gov (NCT03269357). Results: Fourteen intervention clinics enrolled 658 participants from September 2017 to May 2019. Response rate among providers was $88.0 \%$ (55/62) and among participants $97.1 \%$ (639/658). Providers found the intervention to be supportive in their counseling. Each separate part of the intervention package received high ratings from both providers and participants. Participants found the educational video and the effectiveness chart to be more helpful than the box of contraceptive models in their contraceptive choice. Providers reported the time taken to complete the intervention

Abbreviations: CC, contraceptive counselling; HCP, healthcare providers; IQR, interquartile range; IUD, intrauterine device; LARC, long-acting reversible contraceptive; SARC, short-acting reversible contraception.

This is an open access article under the terms of the Creative Commons Attribution-NonCommercial-NoDerivs License, which permits use and distribution in any medium, provided the original work is properly cited, the use is non-commercial and no modifications or adaptations are made.

(C) 2021 The Authors. Acta Obstetricia et Gynecologica Scandinavica published by John Wiley \& Sons Ltd on behalf of Nordic Federation of Societies of Obstetrics and Gynecology (NFOG) 
outside the study to be time-neutral to standard counseling, and most providers wished to continue to use all parts of the intervention package.

Conclusions: The intervention of structured contraceptive counseling had high provider and participant satisfaction. The structured counseling package could be used in several clinical settings to improve quality in contraceptive counseling and to enhance informed decision making about use of contraceptive methods.

\section{KEYWORDS}

choice, contraception, contraceptive counseling, contraceptive implant, intrauterine device, long-acting reversible contraceptives

\section{1 | INTRODUCTION}

Unmet need of contraception and subsequent unintended pregnancy are major reproductive health problems, resulting in high numbers of abortions and high costs for individuals and societies worldwide. The proportion of couples using modern methods for contraception is a key indicator used to measure improvements in access to reproductive health. ${ }^{1}$ Long-acting reversible contraception (LARC) includes intrauterine devices (IUDs) and contraceptive implants. These methods have higher contraceptive effectiveness than user-dependent methods such as pills, patches or rings. ${ }^{2}$ The worldwide estimated use of intrauterine devices is $14 \%$, and despite LARCs being the most effective contraceptive methods available, the estimated use of implants is a mere $0.7 \%{ }^{3}$ Increasing the use of LARCs has been promoted as a means to lower the numbers of unintended pregnancies and abortions and thus improve reproductive health. ${ }^{4,5}$ Interventions to remove financial barriers and to in crease method-specific knowledge with emphasis on contraceptive effectiveness lead to higher use of LARCs and lower number of unintended pregnancies and abortions. ${ }^{6-8}$

In Sweden, contraceptive counseling (CC) is mainly provided by nurse-midwives during 5 - to 30 -minute visits. CC is free of charge and women have access to the full range of contraceptive methods. In addition, most contraceptives are subsidized up to the age of 26 , with a maximum cost of approximately 10 Euros yearly for any contraceptive method. Despite this, a national Swedish survey conducted in 2017 among women of reproductive age, showed an unmet need of contraception of $15 \%$, and a current LARC use of $30.9 \%{ }^{9}$ Among countries with robust data collection, Sweden has a high abortion rate $^{10}$ and $45 \%$ of abortions are requested by women who have experienced a previous abortion. ${ }^{11}$ The current model for CC is provider-dependent and national guidelines for counseling are lacking. It has been shown that Swedish women have low method-specific knowledge among contraceptive users as well as non-users. ${ }^{12}$ This study aimed to evaluate provider and participant satisfaction with the intervention package used in a cluster randomized trial in abortion, youth and maternal health clinics, and to explore whether satisfaction differs between providers and participants or between clinic types. The purpose of the cluster randomized trial was to evaluate

\section{Key message}

The intervention of structured contraceptive counseling is time neutral to standard counseling, has high provider and patient satisfaction and supports contraceptive counseling and choice. Thus, it improves quality of care and enhance informed decision making about use of contraceptive methods

the effects of structured CC on LARC choice and initiation, and pregnancies. $^{13}$

\section{2 | MATERIAL AND METHODS}

A cross-sectional study was performed on participants and healthcare providers (HCPs) in the intervention group of a cluster randomized trial (the LOWE trial) among clinics providing CC in the Stockholm region of Sweden. An open invitation was sent to abortion, youth and maternal health clinics, and those accepting participation were randomized in a 1:1 allocation ratio to give either structured CC (intervention) or to provide their current standard CC (control). Before study start, HCPs at intervention clinics were invited to participate in a 3-hour lecture/discussion on updates in contraception to increase their method-specific knowledge. They were also introduced to all parts of the intervention package. The intervention package consisted of:

I an educational video, available in Swedish with English subtitles, to be seen by the participant prior to the CC, either on a private smartphone in the waiting room or on a screen provided by the clinic;

II four key questions (Data S1) to be asked by the HCP at the counseling visit, followed by

III a modified tiered chart ${ }^{2,14}$ presenting method-specific numbers of pregnancies among 10000 users in a year and percentage (\%) of effectiveness in typical use (Data S2); 
IV a toolbox with natural size models of available contraceptive methods (Data S3).

The intervention is described in more detail elsewhere. ${ }^{13}$ Patients seeking CC or abortion care including CC from any participating clinic received oral and written information about the study and signed informed consent to participate prior to inclusion. They then received structured or standard CC depending on the allocation of the clinic they visited. Inclusion criteria included: ability to read Swedish or English (or receive study information by the help of an interpreter), being $\geq 18$ years of age, and sexually active or planning to be sexually active within 6 months and without a wish to conceive. Exclusion criteria included: sterilized patient or partner, and solely other indication for use of contraception besides pregnancy prevention. All HCPs in the study were nurse-midwives or gynecologists who regularly provided CC. Clinics received a small financial compensation of $€ 2$ for each recruited participant. All CC was free of charge according to routine care. Study subjects received no compensation for participating and had to cover any expenses for their contraceptive method of choice.

The primary outcome of the main trial selected a priori, was the effect of the intervention on LARC choice. This study analyses provider and participant satisfaction with the different parts of the intervention package. For each study participant, the HCPs completed an electronic survey with general questions on the participants' reproductive history, previous or current contraceptive use and contraceptive method chosen. Immediately after the CC, without the presence of the HCP, the participants completed a survey on demographics and baseline characteristics and satisfaction with part I, III and IV of the intervention package. Participants were not aware of what the full intervention package was comprised of, and hence were not asked to rate the key questions or their satisfaction with the intervention as a whole. After the recruitment period, an electronic survey was sent to the HCPs that provided CC at the intervention clinics to collect data on their age and work experience with CC. HCPs were also asked to assess their satisfaction with all parts of the intervention package and whether they would like to continue to use it. Additionally, HCPs were asked to estimate time consumption of providing the intervention outside the study compared with the current standard CC in terms of saving or adding 0-15 minutes on a slider, and to rate their satisfaction with all aspects of the intervention as a whole.

\section{1 | Statistical analyses}

No power calculation was made for the outcomes presented in this study. The sample size was calculated for the primary outcome of the main trial, ${ }^{13}$ resulting in an aim to include 28 clinics and a total of 1200 study participants. Data collected for this study are from HCPs finishing the post-study survey and participants receiving $\mathrm{CC}$ at intervention clinics in the main trial. Proportions were analyzed using Chisquare or Fisher's Exact test as appropriate. The limit of significance was set at a two-sided $p<0.05$. HCP estimates on time consumption with providing the intervention were calculated with nonparametric one-sample Kolmogorov-Smirnov test and presented as median minutes with interquartile range. Analyses were made using IBM ${ }^{\circledR}$ SPSS $^{\circledR}$ Statistics version 27 (IBM Corp., Armonk, NY, USA).

\section{2 | Ethical approval}

The protocol was designed according to existing legal regulations and in accordance with Good Clinical Practice in the conduct of clinical trials and the Declaration of Helsinki. Ethical approval was given on 29 March 2017 by the regional ethics committee in Stockholm, number 2017/525-31/4. The study was registered at Clinicaltrials. gov 25 August 2017, \#NCT03269357, https://clinicaltrials.gov, initial participant enrollment 1 September 2017.

\section{3 | RESULTS}

Of 33 randomized clinics, 14 of those allocated to intervention provided the study intervention in CC. Participants were recruited from September 2017 to May 2019. In total, 62 HCPs gave CC according to the intervention, of which 55 (88.0\%) completed the electronic survey. Of 1085 eligible patients at intervention clinics, 669 were enrolled in the main trial and 658 received the intervention. The flow of clinics, HCPs and participants are described in Figure 1. Baseline characteristics of HCPs and participants are presented in Tables 1 and 2. A higher proportion of the providers found the effectiveness chart (52/55 [94.5\%]) and the box of contraceptive models (50/55 [90.1\%]) to be supportive in CC compared with the proportion of the participants who found the chart (357/639 [55.9\%]) and the box (328/639 [51.3\%]) to be supportive in contraceptive choice $(p<0.001)$. All provider and participant satisfaction with the intervention material are presented in Table 3.

The use of an educational video and key questions was new for 92.7\% (52/55) and $49.1 \%(27 / 55)$ of the HCPs, respectively. Most HCPs had previously used a different effectiveness chart $(76.4 \%$, $42 / 55)$ and demonstration of contraceptive models in their CC $(81.8 \%$, $45 / 55)$. There was a difference in overall satisfaction rates among HCPs related to clinic type, with all HCPs rating it as "poor" $(n=1)$ or "no opinion" ( $n=4)$ working at maternal health clinics ( $p=0.025)$. Among those HCPs, three stated that the intervention was similar to their standard counseling routine, one stated it added time and one provided no comment. In total, there was no correlation between $\mathrm{HCPs}$ being less satisfied and those considering the intervention to consume extra time. A majority of the HCPs stated that they would like to continue to use all parts of the intervention package (Figure 2).

Among the participants, 20.2\% (129/639) reported that they did not know what contraceptive method they intended to use prior to receiving CC. Most of these participants found the intervention parts to be supportive in contraceptive choice-77.5\% (100/129) for the educational video, $63.5 \%(80 / 126)$ for the effectiveness chart, and $62.7 \%(79 / 126)$ for the box of contraceptive models. A higher proportion of women who changed method from their intended 


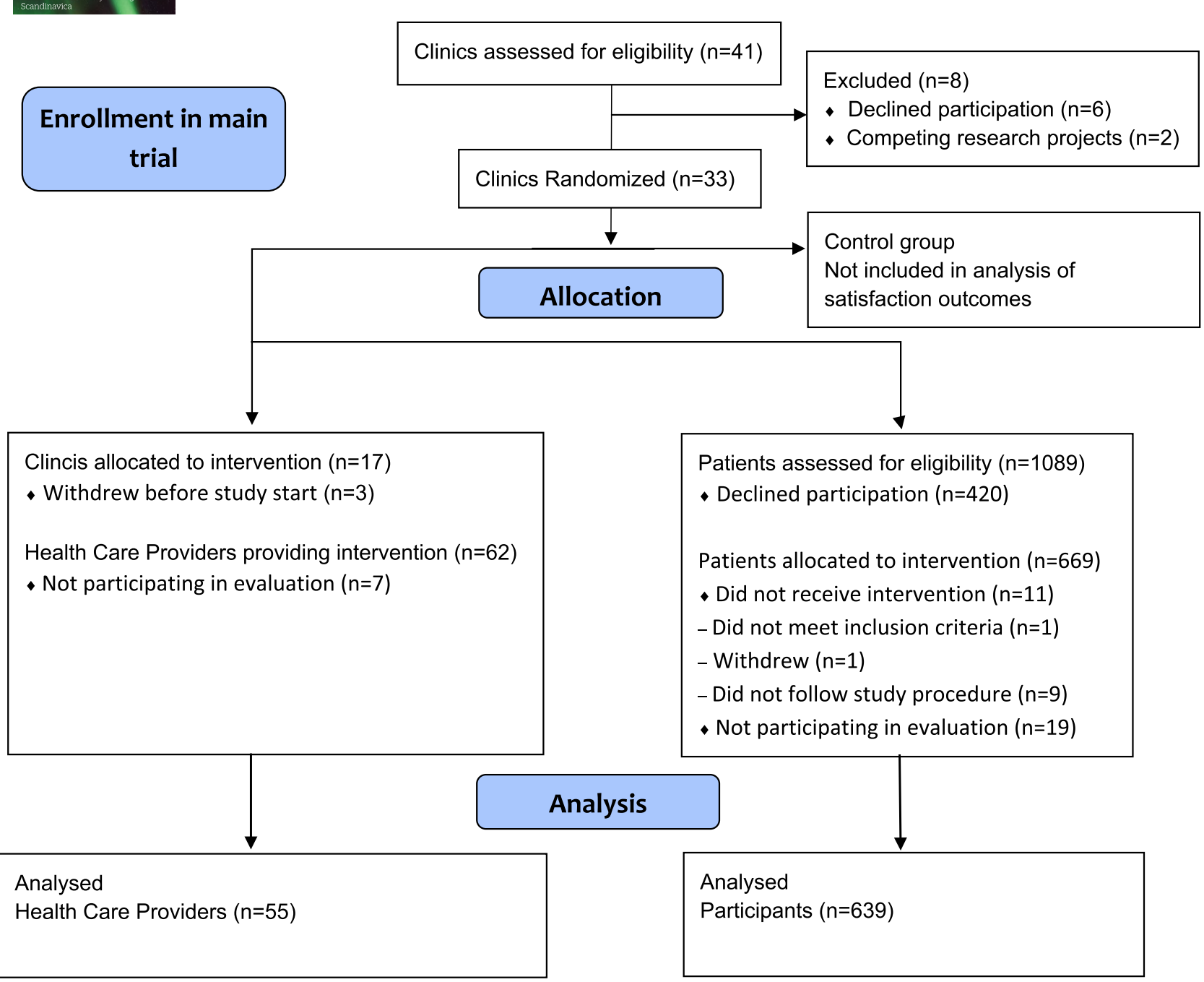

FIGURE 1 Flowchart of clinics, healthcare providers and participants [Color figure can be viewed at wileyonlinelibrary.com]

method found the educational video supportive in their contraceptive choice compared with those who remained with their intended method (60.5\% [196/324] vs 39.5\% [128/324], $p<0.001)$. Similar effects were seen for the effectiveness chart $(60.9 \%$ [165/271] vs $39.1 \%$ [106/271], $p<0.001)$, and the box of contraceptive models (58.2\% [142/244] vs 41.8\% [102/244], $p<0.001)$. Younger participants found the intervention parts to be supportive in contraceptive choice to a higher extent compared with older participants (Figure 3.), and a higher proportion of the participants from abortion and youth clinics than from maternal health clinics stated that the intervention parts were supportive in contraceptive choice (Figure 4).

Intervention adherence was reported by HCPs to be $95.9 \%$ (613/639). Among the 20 CC sessions in which the HCPs reported that the intervention was not fully delivered, 13/20 did not include all the key questions, 13/20 did not include the box of contraceptive models, and six $(6 / 20)$ did not include the effectiveness chart. Stated reasons for not adhering to the intervention was time limitation ( $n=4)$, forgot a specific part $(n=5)$, the patient had already decided on contraceptive method and was not open to further counseling $(n=6)$, the patient did not say they were participating in the study $(n=2)$ and other non-specified reasons $(n=1)$. Participants reported that they were not shown the effectiveness chart or the box of contraceptive models in 10.3\% (66/638) and $19.1 \%(122 / 639)$ of cases, respectively (Table 3). A higher proportion of the participants from maternal health clinics $(48 / 253$ [19.0\%]) reported that they were not shown the effectiveness chart compared with participants from abortion (6/110 [5.5\%]) and youth clinics (12/276 [4.3\%], $p<0.001)$. Similar differences were seen for the box of contraceptive models, which was reported as not shown by $98 / 253$ (38.7\%), 8/110 (7.3\%), and 16/276 (5.8\%) of participants from maternal health clinics, abortion clinics and youth clinics, respectively ( $p<0.001)$.

HCPs estimated time consumption in minutes for providing the intervention outside the study to be time-neutral compared with their current standard CC (median 0, interquartile range [IQR] -5 to 5). A total of $70.9 \%(39 / 55)$ of HCPs believed that the intervention affected the participant's contraceptive choice to a large extent and $18.2 \%(10 / 55)$ to some extent.

\section{4 | DISCUSSION}

We established high satisfaction with our structured CC from both an HCP and participant perspective. The vast majority of HCPs 
TABLE 1 Baseline characteristics of healthcare providers $(n=55)$

\begin{tabular}{|c|c|}
\hline \multicolumn{2}{|l|}{ Age group (years) } \\
\hline $20-29$ & $2(3.6)$ \\
\hline $30-39$ & $10(18.2)$ \\
\hline $40-49$ & $21(38.2)$ \\
\hline $50-59$ & $10(18.2)$ \\
\hline$\geq 60$ & $12(21.8)$ \\
\hline \multicolumn{2}{|l|}{ Clinic type } \\
\hline Abortion clinic & $11(20.0)$ \\
\hline Youth clinic & $19(34.5)$ \\
\hline Maternal health clinic & $25(45.5)$ \\
\hline \multicolumn{2}{|l|}{ Occupation } \\
\hline Medical doctor & $4(7.3)$ \\
\hline Nurse-midwife & $51(92.7)$ \\
\hline \multicolumn{2}{|c|}{ Experience within occupation (years) } \\
\hline $1-2$ & $5(9.1)$ \\
\hline $3-4$ & $1(1.8)$ \\
\hline $5-9$ & $15(27.3)$ \\
\hline $10-14$ & $9(16.4)$ \\
\hline $15-19$ & $5(9.1)$ \\
\hline$\geq 20$ & $20(36.4)$ \\
\hline \multicolumn{2}{|c|}{ Experience of contraceptive counseling (years) } \\
\hline $0-2$ & $10(18.2)$ \\
\hline $3-4$ & $7(12.7)$ \\
\hline $5-9$ & $11(20.0)$ \\
\hline $10-14$ & $10(18.2)$ \\
\hline $15-19$ & $9(16.4)$ \\
\hline$\geq 20$ & $8(14.5)$ \\
\hline
\end{tabular}

Data are $n(\%)$.

found the different parts of the intervention package to be helpful tools in their provision of CC (video $74.5 \%$, chart $94.5 \%$, models $90.1 \%$, key questions $74.5 \%$ ) and were of the opinion that the intervention affected the contraceptive choice of the participants. Furthermore, most of the participants found that the intervention parts (video $67.4 \%$, chart $55.9 \%$, models $51.3 \%$ ) supported them in their choice of contraceptive method.

The strength of the cluster-randomized design of the main trial is inherent to this study since it reduces risk of selections bias and adds to external validity of the results. Another strength is the use of multimodal tools (audio, visual, written) to aid a diverse group of CC participants of different background, knowledge and experience to make an informed decision of contraceptive method use. This is reflected in the high satisfaction rates in this study. Another strength of the analysis is the high response rate from participants and HCPs which ensures a reliable evaluation of the different intervention parts and the intervention as a whole. The intervention reached a large number of participants which contributes to the external validity of the results. A limitation of the intervention is that the educational video
TABLE 2 Baseline characteristics of participants $(n=639)$

\begin{tabular}{lc} 
Age, median (IQR) & $24(20-29)$ \\
Clinic type & $111(16.9)$ \\
Abortion clinic & $283(43.0)$ \\
Youth clinic & $264(40.1)$ \\
Maternal health clinic & \\
\hline Highest completed education, $n=639(11.0)$ \\
Primary school & $70(51.5)$ \\
Secondary school & $1329(37.4)$ \\
College/University & $10.2)$ \\
Unknown & \\
Reproductive history & $218(34.1)$ \\
Ever pregnant, $n=637$ & $521(81.5)$ \\
Nulliparous, $n=635$ & $159(25.0)$ \\
Abortion, $n=636$ & \\
\hline Current contraception $n=635$ & $29(4.5)$ \\
LARC & $201(31.5)$ \\
SARC & $86(13.0)$ \\
Other & \\
None & $322(50.4)$ \\
\hline
\end{tabular}

Data are $n(\%)$.

IQR, interquartile range; LARC, long-acting reversible contraception; SARC, short-acting reversible contraception.

antrauterine device or subdermal implant.

${ }^{b}$ Combined pill, progestin only pill, contraceptive injection, transdermal patch, vaginal ring.

${ }^{\mathrm{C}}$ Condom, diaphragm, smartphone application, fertility awareness methods.

was only available in Swedish with English subtitles. Participants with insufficient Swedish or English knowledge had to rely on professional interpreters to understand the intervention material fully.

Previously, a discrepancy between what women need and want, and what is provided in CC has been shown. ${ }^{15}$ In an extensive online survey of 6027 women, it was found that $60 \%$ of women were interested in receiving more information about all methods and $73 \%$ of women said they would consider LARC if they received more comprehensive information. ${ }^{16}$ Trials estimating effects of structured CC on contraceptive choice have shown that CC can alter the choice of contraceptive methods. However, for an intervention in personally delivered $\mathrm{CC}$ to be feasible for implementation, the acceptability of the intervention method must be high for HCPs and participants alike. Satisfaction with structured counseling has been shown in a study of counseling on different methods of combined hormonal contraception. A leaflet with information on different combined hormonal contraceptives was used that covered method characteristics, common side effects and contraindications. HCPs used the structured counseling in $86 \%$ of the counseling sessions, and most participants found the information "somewhat" or "very" useful (96\%). ${ }^{17}$ In our study, the intervention package received high satisfaction ratings and was found to be supportive for both HCPs and participants. In addition, the intervention resulted in higher LARC uptake compared with 
scandinater

\section{Healthcare providers \\ Participants \\ $n=55$ \\ $n=639$ \\ $p$-value}

\section{Educational video}

$$
\text { Very good }
$$

$25(45.5)$

27 (49.1)

Good

$3(5.5)$

No opinion

Poor

Very poor

Video was supportive in contraceptive counseling/ choice

\section{Effectiveness chart}

Very good

32 (58.2)

$0(0)$

$0(0)$

41(74.5)

20 (36.4)

2 (3.6)

1 (1.8)

$0(0)$

$6\left(0.9^{\mathrm{a}}\right)$

$52(94.5)$

Chart was supportive in

contraceptive counseling/

choice

Box of contraceptive models

\section{Very good \\ Good}

No opinion

Poor

Very poor

Did not show/not shown

Models were supportive in contraceptive counseling/ choice
$378(59.2)$

$230(36.0)$

$28(4.4)$

$3(0.5)$

$0(0)$

$431(67.4)$

0.28
TABLE 3 User satisfaction with intervention parts by healthcare providers and participants

$\begin{array}{lc}4 \text { key-questions } & \\ \text { Very good } & 17(30.1) \\ \text { Good } & 23(41.8) \\ \text { No opinion } & 11(20.0) \\ \text { Poor } & 4(7.3) \\ \text { Very poor } & 0(0) \\ \text { Questions were supportive in } & 41(74.5) \\ \quad \text { contraceptive counseling } & \\ \text { Overall satisfaction with } & \\ \text { intervention material } & \\ \text { Very good } & 21(38.2) \\ \text { Good } & 29(52.7) \\ \text { No opinion } & 4(7.3) \\ \text { Poor } & 1(1.8) \\ \text { Very poor } & 0(0)\end{array}$

Data are $\mathrm{n}(\%)$. Significance level calculated with Chi-square and Fisher's Exact tests where appropriate.

NA, not applicable.

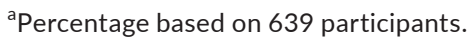

${ }^{\mathrm{b}}$ Rated only by healthcare providers. 
FIGURE 2 Percentage of healthcare providers (HCPs) wanting to continue to use the intervention, by intervention parts [Color figure can be viewed at wileyonlinelibrary.com]
FIGURE 3 Percentage of participants, in each age group who found the intervention parts to be supportive in contraceptive choice, by intervention parts. ${ }^{*} p<0.001$. Significance levels calculated with Chi-Square tests [Color figure can be viewed at wileyonlinelibrary. com]
PERCENTAGE OF HCPS WANTING TO CONTINUE TO USE THE INTERVENTION PARTS

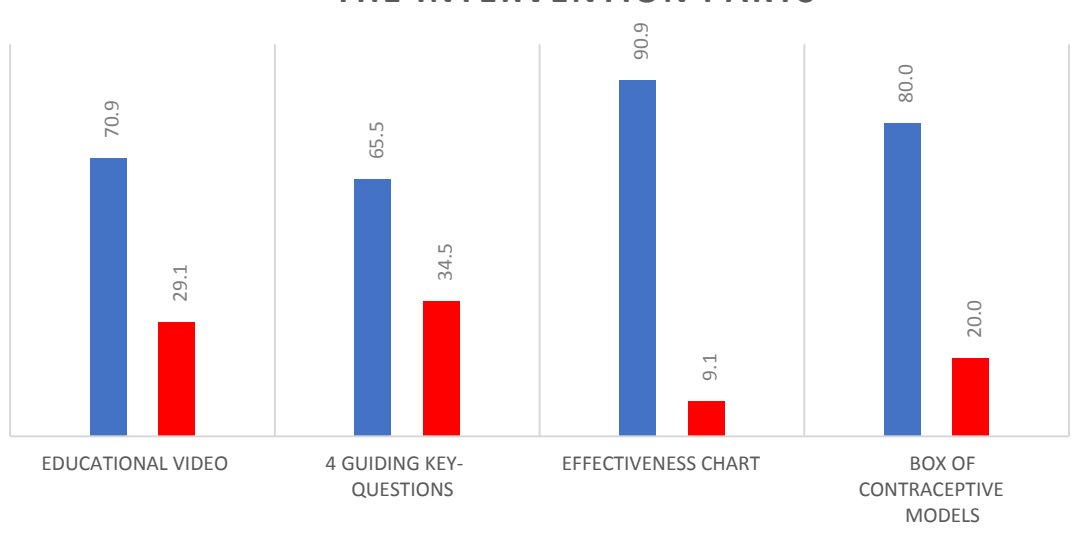

- Yes No

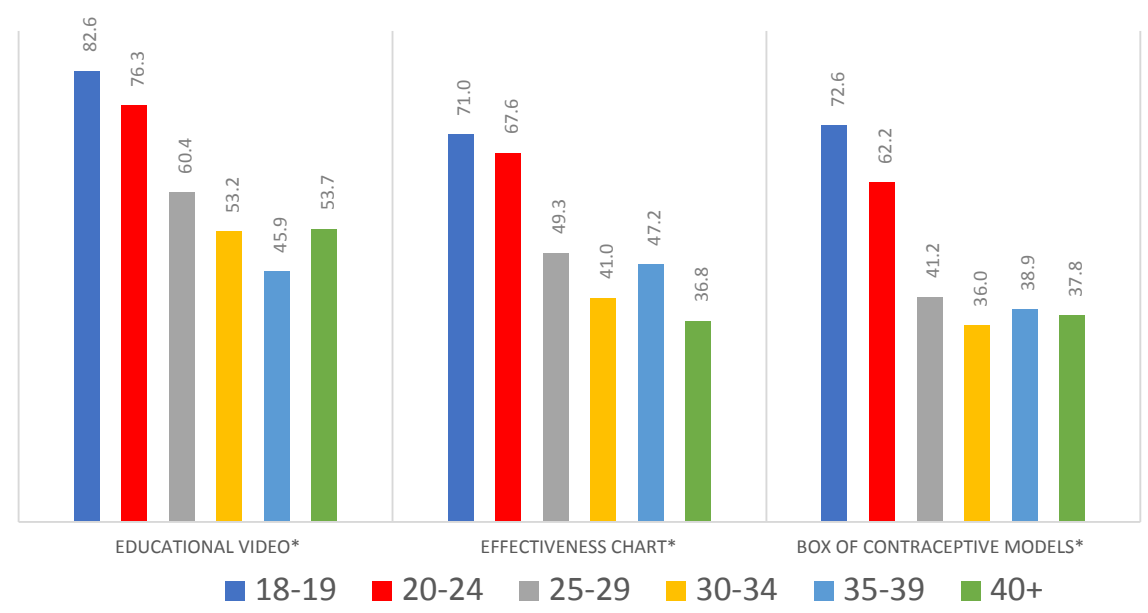

PERCENTAGE OF PARTICIPANTS WHO FOUND INTERVENTION PARTS BEING SUPPORTIVE IN CONTRECEPTIVE CHOICE, BY CLINIC TYPE

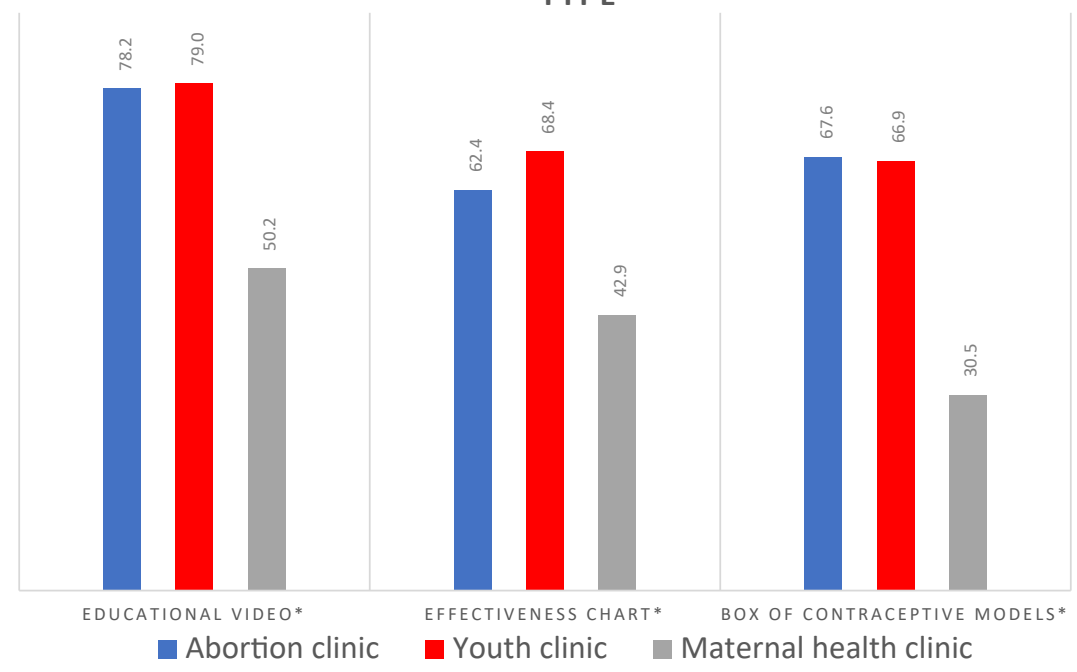

FIGURE 4 Percentage of participants who found the intervention parts to be supportive in contraceptive choice, by clinic type. ${ }^{*} p<0.001$. Significance levels calculated with Chi-Square tests [Color figure can be viewed at wileyonlinelibrary. com]

\section{PARTS BEING SUPPORTIVE IN CONTRACEPTIVE CHOICE, BY AGE GROUP}


routine counseling, and fewer unintended pregnancies were seen in abortion patients at a 12 -month follow-up. ${ }^{13}$ Although a very high proportion of the participants rated the intervention parts as very good or good, we saw that a lower proportion, but still a majority, found the intervention parts to be supportive in contraceptive choice. A possible explanation of this difference can be found in the result that participants receiving their CC in maternal health clinics were less supported by the intervention. This clinic type stands out concerning participants who were never shown the effectiveness chart or the box of contraceptive models, and hence could not have been supported by them. There were also significant differences when comparing the proportions of providers and participants who found the effectiveness chart and the box of contraceptive models to be supportive in $\mathrm{CC}$ and contraceptive choice. The reason behind these results cannot be explained by the current methodology of this study. A possible explanation could be a perception of lack of structure in the regular CC that HCPs were practicing before entering the study, and that the intervention helped solve this issue. Another explanation could be that participants who shifted from their intended contraceptive method were more satisfied with the intervention material than were those who maintained their intended method. Non-shifters probably had already made their decision prior to counseling and hence did not find the material to be supportive in contraceptive choice. The aim of the intervention was to enable the participant to make an informed decision. ${ }^{13}$ An additional question of whether the participants found the intervention to fulfil that purpose would have added value to this evaluation of the intervention. The use of educational videos as an intervention part in CC has been evaluated by other researchers, who found that it increased method specific knowledge ${ }^{18,19}$ and affected contraceptive choice toward a more effective contraceptive method. ${ }^{18,20}$ The use of tiered charts to show contraceptive effectiveness has been shown to increase method-specific knowledge and to increase LARC uptake. ${ }^{21-23}$ The use of contraceptive models in counseling makes women more aware of actual size and route of administration, which might lead to finding contraceptives smaller and less frightening than imagined. ${ }^{24}$ In our intervention, we included multimodal tools to increase knowledge about contraceptive methods and to enable informed decision making about contraceptive choice. Participants who changed contraceptive method from their intended method reported a high satisfaction with the $\mathrm{CC}$ and that the intervention was supportive in their contraceptive choice. Likewise, most participants stating that they did not know what method to use prior to $\mathrm{CC}$, found the intervention to be supportive in their contraceptive choice. We could also show that younger participants were more supported by the intervention. Younger women may have less knowledge and experience with different contraceptive methods and, in addition, are more susceptible to unintended and unwanted pregnancies ${ }^{25}$ and subsequent abortions. ${ }^{26}$

Our intervention aimed at providing comprehensive information on contraceptive methods with emphasis on contraceptive effectiveness. The use of the One Key Question ${ }^{\circledR}$ method, asking women "would you like to become pregnant in the next year?" has been proven to increase the proportion of women receiving $\mathrm{CC}$ within primary care settings in the USA. ${ }^{27}$ In contrast, our intervention included four key questions. Our questions were formulated to make the participant reflect on their reproductive life plan and pregnancy intention. In addition, we included questions on menstrual patterns that aimed to emphasize added health benefits, which could be beneficial for patients suffering from heavy menstrual bleeding, dysmenorrhea or endometriosis.

The time consumption for the intervention if used in clinical practice was estimated by HCPs to be time-neutral compared with current standard counseling, which is important for acceptability and future clinical implementation. The amount of time allocated for each patient is a well-known barrier to provide patient-centered and comprehensive CC. ${ }^{16,28}$ To our knowledge, our intervention package is the first intervention package to comprise a pre-counseling tool (educational video) as well as key questions and in-counseling tools (effectiveness chart and box of contraceptive models). Although satisfaction with the different parts of the intervention could be evaluated, it is not possible to assess which parts of the intervention influenced contraceptive choice. Therefore, the full intervention should be used if implemented in clinical practice.

Given that our intervention can be used without adding extra time for counseling, it could be used as a cost-effective intervention in several clinical settings to improve quality in CC.

\section{5 | CONCLUSION}

A majority of HCPs and participants were satisfied with the intervention and considered it to be supportive in their $\mathrm{CC}$ and contraceptive choice. The intervention was considered time-neutral compared with standard counseling and could be easily introduced into several clinical settings to improve the quality of care regarding CC and to enhance informed decision-making in contraceptive choice.

\section{ACKNOWLEDGMENTS}

The authors wish to thank all HCPs at the study sites for their invaluable help in recruitment of study participants and for their contribution in evaluating the study intervention, Annelie Wikström, RNM for study site attendance, Rebecca Erkenstam and Sophie Baehrendtz, MD Students and Cecilia Arvidson, RNM for contributing to the work with study participant follow-up.

\section{CONFLICT OF INTEREST}

The authors declare no support from any organization for the submitted work. Outside the submitted work, NE reports personal fees from Bayer AG, KEl reports non-financial support from Bayer AG and RemovAid AS, KGD reports other support from RemovAid, personal fees and other support from MSD/Merck, Bayer AG, Gedeon Richter, Mithra, Exeltis, HRA-Pharma, Exelgyn, Campus Pharma, Cirqle, Natural Cycles, MedinCell and Myovant, HKK reports personal fees and other support from Bayer, personal fees from MSD, Exeltis, Mithra, Natural Cycles, Gedeon Richtera and Preglem. IB declares no conflicts of interest. 


\section{AUTHOR CONTRIBUTIONS}

KGD was responsible for the study concept, trial protocol, regulatory approvals and overall for the study conduct and funding. HKK contributed to study design protocol development and in securing funding as well as in supervision of the study. All authors except IB were involved in the development of study tools and recruitment of participating clinics. NE, KEI and IB acquired the data. NE was responsible for data analyses. All authors contributed to the interpretation of the data and manuscript writing.

\section{ORCID}

Niklas Envall (D) https://orcid.org/0000-0003-2813-7642

Karin Emtell Iwarsson (D) https://orcid.org/0000-0003-3659-2637

Isabella Bizjak (D) https://orcid.org/0000-0003-2893-2312

Kristina Gemzell Danielsson (D) https://orcid.

org/0000-0001-6516-1444

Helena Kopp Kallner (D) https://orcid.org/0000-0001-7184-9165

\section{REFERENCES}

1. United Nations, Department of Economic and Social Affairs. Population Division (2019). World Contraceptive Use 2019 (POP/ $\mathrm{DB} / \mathrm{CP} / \mathrm{Rev} 2019)$.

2. Trussell J. Contraceptive failure in the United States. Contraception. 2011;83:397-404.

3. United Nations, Department of Economic and Social Affairs. Population Division (2015). Trends in Contraceptive Use Worldwide 2015.

4. Population Council, International Federation of Gynaecology and Obstetrics (FIGO), and Reproductive Health Supplies Coalition. 2013 Statement from the Bellagio Group on LARCs: Long-Acting Reversible Contraception in the Context of Full Access, Full Choice. 18 December 2013. Available at www.popcouncil.org/ pdfs/2013RH_BellagioConsensus.pdf

5. Curtis KM, Tepper NK, Jatlaoui TC, et al. U.S. Medical eligibility criteria for contraceptive use, 2016. MMWR. Recommend Rep. 2016;65(3):1-103.

6. Peipert JF, Madden T, Allsworth JE, Secura GM. Preventing unintended pregnancies by providing no-cost contraception. Obstet Gynecol. 2012;120:1291-1297.

7. Gyllenberg F, Juselius M, Gissler M, Heikinheimo O. Long-acting reversible contraception free of charge, method initiation, and abortion rates in Finland. Am J Public Health. 2018;108:538-543.

8. Madden T, Mullersman JL, Omvig KJ, Secura GM, Peipert JF. Structured contraceptive counseling provided by the Contraceptive CHOICE Project. Contraception. 2013;88:243-249.

9. Hellstrom A, Gemzell Danielsson K, Kopp Kallner H. Trends in use and attitudes towards contraception in Sweden: results of a nationwide survey. Eur J Contracept Reprod Health Care. 2019;24:154-160.

10. Singh S, Remes L, Sedgh G, Kwok L, Onda T. Abortion Worldwide 2017: Uneven Progress and Unequal Access. Guttmacher Institute; 2018.

11. Socialstyrelsen. Statistik om aborter 2018 [Statistics on abortions 2018]. In Swedish; 2019. https://www.socialstyrelsen.se/

12. Kopp Kallner H, Thunell L, Brynhildsen J, Lindeberg M, Gemzell DK. Use of contraception and attitudes towards contraceptive use in Swedish Women-a nationwide survey. PLoS One. 2015;10:e0125990.

13. Emtell Iwarsson K, Envall N, Bizjak I, Bring J, Kopp Kallner H, Gemzell-Danielsson K. Increasing uptake of long-acting reversible contraception with structured contraceptive counselling: cluster randomised controlled trial (the LOWE trial). BJOG. 2021;128:1546-1554.
14. Steiner MJ, Trussell J, Johnson S. Communicating contraceptive effectiveness: an updated counseling chart. Am J Obstet Gynecol. 2007;197:118.

15. Dehlendorf C, Levy K, Kelley A, Grumbach K, Steinauer J. Women's preferences for contraceptive counseling and decision making. Contraception. 2013;88(2):250-256.

16. Merki-Feld GS, Caetano C, Porz TC, Bitzer J. Are there unmet needs in contraceptive counselling and choice? Findings of the European TANCO Study. Eur J Contracept Reprod Health Care. 2018;23:183-193.

17. Bitzer J, Gemzell-Danielsson K, Roumen F, Marintcheva-Petrova M, van Bakel B, Oddens BJ. The CHOICE study: effect of counselling on the selection of combined hormonal contraceptive methods in 11 countries. Eur J Contracept Reprod Health Care. 2012;17:65-78.

18. Sanders JN, Moran LA, Mullholand M, Torres E, Turok DK. Video counseling about emergency contraception: an observational study. Contraception. 2019;100:54-64.

19. Dineley B, Patel T, Black M, Koziarski R, Lamarche L, Costescu D. Video media in clinic waiting areas increases interest in most effective contraceptive methods. J Obstet Gynaecol Can. 2018;40:1302-1308.

20. Harper CC, Rocca CH, Thompson KM, et al. Reductions in pregnancy rates in the USA with long-acting reversible contraception: a cluster randomised trial. Lancet. 2015;386:562-568.

21. Steiner MJ, Trussell J, Mehta N, Condon S, Subramaniam S, Bourne D. Communicating contraceptive effectiveness: a randomized controlled trial to inform a World Health Organization family planning handbook. Am J Obstet Gynecol. 2006;195:85-91.

22. Lopez LM, Steiner M, Grimes DA, Hilgenberg D, Schulz KF. Strategies for communicating contraceptive effectiveness. Cochrane Database Syst Rev. 2013;30(4):CD006964.

23. Thompson KMJ, Rocca CH, Stern L, et al. Training contraceptive providers to offer intrauterine devices and implants in contraceptive care: a cluster randomized trial. Am J Obstet Gynecol. 2018;218:597.e1-e7.

24. Bharadwaj P, Saxton JC, Mann SN, Jungmann EM, Stephenson JM. What influences young women to choose between the emergency contraceptive pill and an intrauterine device? A qualitative study. Eur J Contracept Reprod Health Care. 2011;16:201-209.

25. Finer LB, Zolna MR. Declines in unintended pregnancy in the United States, 2008-2011. N Engl J Med. 2016;374:843-852.

26. Jerman J, Jones RK, Onda T. Characteristics of US abortion patients in 2014 and changes since 2008. Guttmacher Institute. 2016:1-27.

27. Stulberg DB, Dahlquist IH, Disterhoft J, Bello JK, Hunter MS. Increase in contraceptive counseling by primary care clinicians after implementation of one key question(R) at an Urban Community Health Center. Matern Child Health J. 2019;23:996-1002.

28. Dehlendorf C, Reed R, Fitzpatrick J, Kuppermann M, Steinauer J, Kimport K. A mixed-methods study of provider perspectives on My Birth Control: a contraceptive decision support tool designed to facilitate shared decision making. Contraception. 2019;100:420-423.

\section{SUPPORTING INFORMATION}

Additional Supporting Information may be found online in the Supporting Information section.

How to cite this article: Envall N, Emtell Iwarsson K, Bizjak I, Gemzell Danielsson K, Kopp Kallner H. Evaluation of satisfaction with a model of structured contraceptive counseling: Results from the LOWE trial. Acta Obstet Gynecol Scand. 2021;00:1-9. https://doi.org/10.1111/aogs.14243 